\title{
Development of a machine learning model to predict the risk of late cardiogenic shock in patients with ST-segment elevation myocardial infarction
}

\author{
Zhixun Bai ${ }^{1,2,3 \#}$, Shan Hu${ }^{1,2 \#}$, Yan Wang ${ }^{1,2 \#}$, Wenwen Deng ${ }^{1,2}$, Ning Gu ${ }^{1,2}$, Ranzun Zhao ${ }^{1,2}$, Wei Zhang ${ }^{2}$, \\ Yi Ma ${ }^{2}$, Zhenglong Wang ${ }^{2}$, Zhijiang Liu ${ }^{2}$, Changyin Shen ${ }^{3}$, Bei Shi ${ }^{1,2}$ \\ ${ }^{1}$ College of Medicine, Soochow University, Suzhou, China; ${ }^{2}$ Department of Cardiology, Affiliated Hospital of Zunyi Medical University, Zunyi, \\ China; ${ }^{3}$ Department of Internal Medicine, The Second Affiliated Hospital of Zunyi Medical University, Zunyi, China \\ Contributions: (I) Conception and design: Z Bai; (II) Administrative support: B Shi; (III) Provision of study materials or patients: R Zhao, W Zhang, \\ Y Ma, Z Wang, Z Liu, C Shen; (IV) Collection and assembly of data: W Deng, N Gu; (V) Data analysis and interpretation: Z Bai, S Hu, Y Wang; \\ (VI) Manuscript writing: All authors; (VII) Final approval of manuscript: All authors. \\ \#These authors contributed equally to this work and served as co-first authors. \\ Correspondence to: Bei Shi, MD. Department of Cardiology, Affiliated Hospital of Zunyi Medical University, Zunyi, China. Email: shibei2147@163.com.
}

Background: The in-hospital mortality of patients with ST-segment elevation myocardial infarction (STEMI) increases to more than 50\% following a cardiogenic shock (CS) event. This study highlights the need to consider the risk of delayed calculation in developing in-hospital CS risk models. This report compared the performances of multiple machine learning models and established a late-CS risk nomogram for STEMI patients.

Methods: This study used logistic regression (LR) models, least absolute shrinkage and selection operator (LASSO), support vector regression (SVM), and tree-based ensemble machine learning models [light gradient boosting machine (LightGBM) and extreme gradient boosting (XGBoost)] to predict CS risk in STEMI patients. The models were developed based on 1,598 and 684 STEMI patients in the training and test datasets, respectively. The models were compared based on accuracy, the area under the curve (AUC), recall, precision, and Gini score, and the optimal model was used to develop a late CS risk nomogram. Discrimination, calibration, and the clinical usefulness of the predictive model were assessed using C-index, calibration plotd, and decision curve analyses.

Results: A total of 2282 STEMI patients recruited between January 1, 2016 and May 31, 2020, were included in the complete dataset. The linear models built using LASSO and LR showed the highest overall predictive power, with an average accuracy over 0.93 and an AUC above 0.82 . With a C-index of 0.811 [95\% confidence interval (CI): 0.769-0.853], the LASSO nomogram showed good differentiation and proper calibration. In internal validation tests, a high $\mathrm{C}$-index value of 0.821 was achieved. Decision curve analysis (DCA) and clinical impact curve (CIC) examination showed that compared with the previous score-based models, the LASSO model showed superior clinical relevance.

Conclusions: In this study, five machine learning methods were developed for in-hospital CS prediction. The LASSO model showed the best predictive performance. This nomogram could provide an accurate prognostic prediction for CS risk in patients with STEMI.

Keywords: Machine learning; cardiogenic shock (CS); ST-segment elevation myocardial infarction (STEMI); least absolute shrinkage and selection operator (LASSO)

Submitted May 18, 2021. Accepted for publication Jul 02, 2021.

doi: 10.21037/atm-21-2905

View this article at: https://dx.doi.org/10.21037/atm-21-2905

^ ORCID: 0000-0003-4352-0658. 


\section{Introduction}

Cardiogenic shock (CS) is the most common cause of inhospital death in patients with acute myocardial infarction (AMI), occurring in approximately $5-10 \%$ of patients. Up to $70 \%$ of CS cases can be attributed to AMI $(1,2)$. Mortality among patients with AMI-complicated CS remains high despite early revascularization. ST-segment elevation myocardial infarction (STEMI) is the most severe type of AMI, with poor prognosis and high mortality $(3,4)$. In China, a recent survey suggests that admission rates (per $100,000)$ for cases of STEMI has increased approximately 4-fold, from 4.6 to 18.0 in men, and 1.9 to 8.0 in women, between 2001 and 2011 (5).

Studies have suggested that the in-hospital mortality of STEMI patients increases to more than $50 \%$ after a secondary CS event, especially within 30 days (6). In addition, STEMI-related complications represent a huge medical and economic burden. Some believe that the high mortality rate and the high incidence of complications in STEMI patients are related to the lack of early effective prevention and intervention measures (7). The lag in intervention has been attributed to delayed first medical contact (FMC), the lack of valuable predictive markers, and the inability of traditional scoring scales to provide accurate predictions. Considering the risk of CS and its many related risk factors, an accurate clinical prediction tool must be developed to accurately predict the occurrence of CS. The main risk factors resulting in the death of STEMI patients including older age, previous MI, renal dysfunction, cardiogenic shock, anterior MI, out-of-hospital cardiac arrest, non-reperfused patients. Several risk scores have been developed in the last 20 years to stratify patients hospitalized with acute coronary syndromes (ACS) (8). The most commonly used score is the Global Registry of Acute Coronary Events (GRACE) risk model, which uses eight variables and applies to the entire spectrum of ACS (9). The Brittany Regional Infarction Observatory (ORBI) study provided a scoring system to identify STEMI patients with impending CS following percutaneous coronary intervention (PCI) (10). However, these risk scores were developed by traditional regression methods, and the use of procedural characteristics in the prediction model may cause a delay in calculating the risk while awaiting the results. Thus, in many cases, the shock develops before the risk can be assessed.

Machine learning is a multidisciplinary field involving artificial intelligence, computational complexity theory, that can be characterized by system self-improvement. Based on machine learning methods and admission variables, a nomogram model may improve performance for STEMI patients who may have CS after admission. To the best of our knowledge, this is the first study to use machine learning algorithms to establish an accurate and easy method for predicting the occurrence of STEMI using readily available features on admission. Different machine learning methods for late CS prediction were compared. We present the following article in accordance with the TRIPOD reporting checklist (11) (available at https:// dx.doi.org/10.21037/atm-21-2905)

\section{Methods}

\section{Patients}

All procedures performed in this study involving human participants were in accordance with the Declaration of Helsinki (as revised in 2013). Research approval was obtained from the Ethics Committee of the Affiliated Hospital of Zunyi Medical University (approval No. KLL[2020]0144). Patient written informed consent was waived due to the retrospective nature of the study. Patients were recruited from the Affiliated Hospital of Zunyi Medical University between January 2016 and May 2020 (Figure 1) according to the STEMI criteria of the European Society of Cardiology (ESC) [2017] (8).

\section{Outcomes}

The primary outcome was in-hospital CS defined as systolic blood pressure $\leq 90 \mathrm{mmHg}$ for more than 30 minutes following the exclusion of hypovolaemia, with clinical evidence of hypoperfusion (cool extremities or a urine output of $<30 \mathrm{~mL} / \mathrm{h}$ and a heart rate $\geq 60$ beats $/ \mathrm{min}$ ) or the requirement for mechanical left ventricular support to correct the condition (12). Late CS was defined as CS developed in the ward. Patients with CS on admission were excluded.

\section{Candidate predictors}

Demographic data, disease, electrocardiographic data, laboratory parameters on admission, and in-hospital events were collated from the patient's medical records. Baseline characteristics, demographics (age and gender), risk factors (hypertension, diabetes, current smoking, family history), 


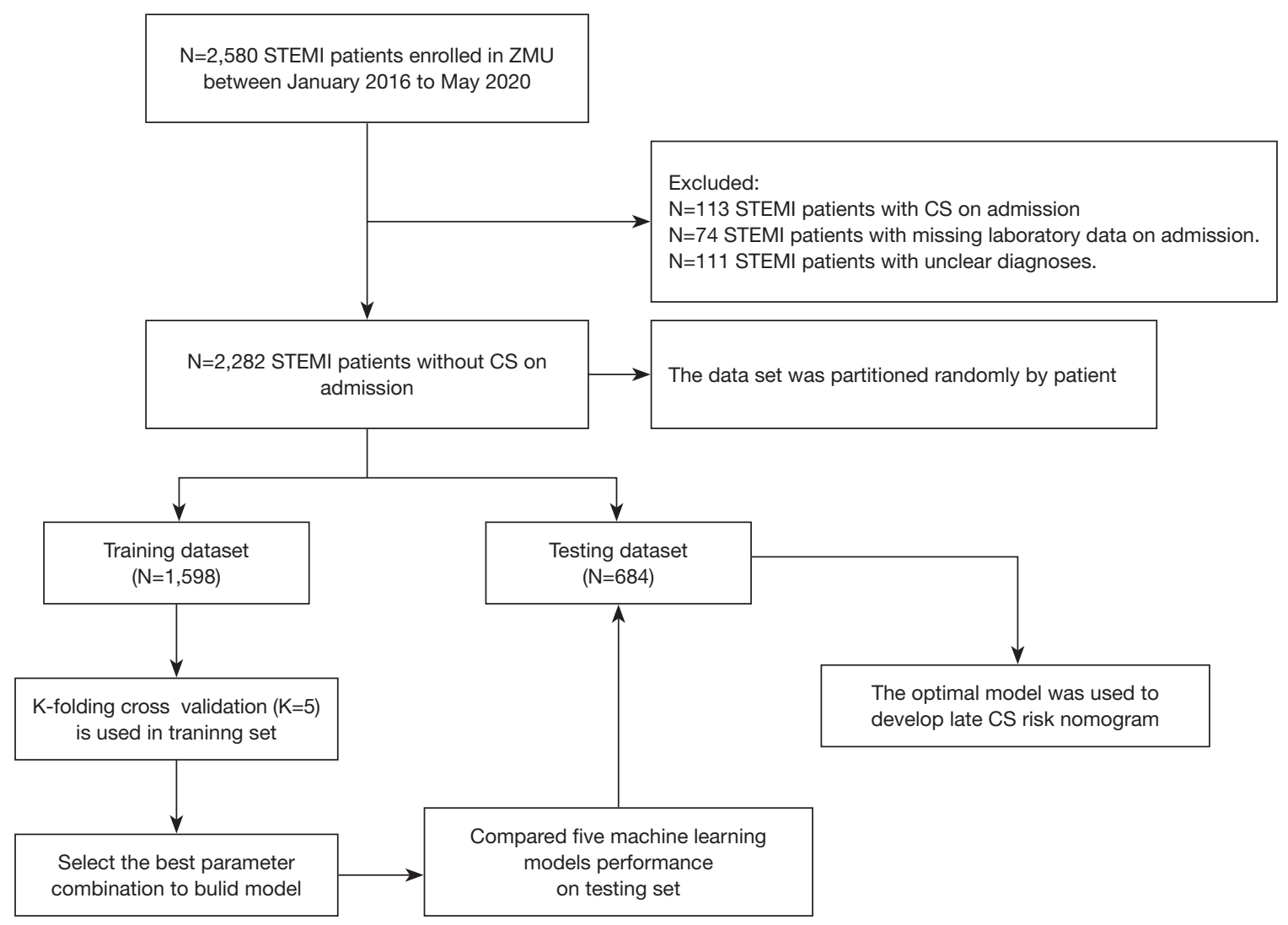

Figure 1 A flow diagram showing the study process. Training and test dataset generation, model training and performance evaluation. A total of 2,282 patients were recruited in the current study. The data were preprocessed and randomly divided into a training set (70\%) and a test set (30\%), maintaining similar proportions for the two classes proportions in each set. In the training set, $\mathrm{k}$-fold cross-validation ( $\mathrm{k}=5$ ) was used. STEMI, ST-segment elevation myocardial infarction; CS, cardiogenic shock.

non-weekday admission (NWDS), delay (defined as patient FMC $>12$ hours), medical history [previous stroke, previous chronic kidney disease (CKD)], and electrocardiogram (ECG) findings (inferior, anterior, right ventricular, other) were all recorded from our electronic database. Patients with an estimated glomerular filtration rate (eGFR) $<60 \mathrm{~mL} / \mathrm{min} / 1.73 \mathrm{~m}^{2}$ for more than three months were defined as having CKD, where the eGFR was calculated for all patients using the Chronic Kidney Disease Epidemiology Collaboration (CKD-EPI) creatinine equation (13). The dataset was randomly partitioned into the training set and a test set. Based on TRIPOD reporting guidelines, the rule of thumb for sample size is to have at least 10 outcome events per variable (EPV). Assessment of predictors in our study has be done without knowledge of the participant's outcome. A single investigator assessed all demographic information and clinical data and was blinded to the outcome.

\section{Missing data}

Complete case data were collected from the electronic health records (EHRs) and analyzed. All variables can be queried in the EHRs. Some patients were excluded as they refused the candidate predictor laboratory test.

\section{Statistical analysis}

CS risk prediction models were bases on five machine learning methods, namely, logistic regression (LR), light gradient boosting machine (LightGBM), extreme gradient boosting (XGBoost), support vector machine (SVM), and least absolute shrinkage and selection operator (LASSO) regression. All numbers listed initially with each hyperparameter were considered default values. Continuous variables are presented as medians, and categorical variables are presented as absolute counts and percentages (n, \%). 
To determine the variables associated with CS risk and to create a predictive model using these variables, machine learning methods and LR analyses were employed. The discriminative abilities of the machine learning models were evaluated and the hyperparameters were tuned by calculating the area under the curve (AUC), accuracy, recall, precision, and the Gini coefficient. The optimal model was used to determine the best combination of variables and develop a nomogram. The variables selected by the best model were manually removed by trial and error to obtain as accurate a model as possible with the smallest number of variables. Five-fold cross-validation $(\mathrm{CV})$ with the training cohort was employed with different randomization schemes. A multivariable LR model was created for comparison by using the variables identified by the best model. Calibration curves were determined as for the best model. These characteristics are described as odds ratios (OR) with $95 \%$ confidence intervals (CI) and P values. The CI of Harrell's concordance index was obtained by creating 1,000 bootstrap samples from the entire dataset and replicating the estimation process. The calibration curve was used to analyze the agreement between the nomogram and ideal observations. Decision curve analysis (DCA) was conducted to assess the clinical usefulness of the predictive nomograms by quantifying the net benefits at different threshold probabilities. All analyses were conducted with the statistical packages $\mathrm{R}$ version 4.0.2 and Python 3.7.

\section{Results}

\section{Model selection}

In this study, 1,598 and 684 STEMI patients, including 117 and 53 patients with CS records, were included in the training and test datasets, respectively (Table 1). The distributions of these variables are shown in Table S1 (variable correlation heatmap in Figure S1). Among the individuals included in the training dataset, the median age was 64 years [interquartile range (IQR) 53, 73], 75\% were male, and $9 \%$ had CKD. Table 2 presents the accuracy, AUC, recall, precision, and Gini coefficient values of the logistic, LASSO, LightGBM, XGBoost, and SVM models with the training and test datasets in predicting CS. The accuracy and AUC with the test dataset show the performance of the developed models. The linear models, LASSO and LR, showed the highest overall predictive power, with average accuracy and AUC above 0.93 and 0.82 , respectively, and similar models produced similar results.
However, the feature selection method was not applied in the logistic model, therefore, the LASSO regression method with regularization was adopted. Generally, the LightGBM and XGBoost algorithms showed better performance with the training and test datasets. However, the LASSO model had the highest AUC and accuracy (0.822 and 0.931 , respectively) with the test dataset (Figure S2). This indicated that LightGBM and XGBoost may encounter more serious overfitting problems compared to other algorithms. In binary classification, LASSO regression may have greater accuracy and demonstrate more convenient advantages compared to other algorithms (Figures S3,S4).

\section{Predictors of in-hospital CS by the LASSO model selection}

Of the 33 initial demographic, disease, and lifestyle characteristics, 8 potential indicators based on 2,282 patients were ultimately retained and included in the LASSO regression model with nonzero coefficients (Figure S5). These characteristics included age, CKD, shock index (SI, define as ratio of HR to SBP), delay, white blood cell (WBC) count, hemoglobin (HB), aspartate aminotransferase (AST), and lactic acid dehydrogenase (LDH) (Table S2).

\section{LASSO risk nomogram model building}

A model that incorporated the above independent predictors was developed and presented as a nomogram (Figure 2A). The nomogram results for patient \#6 are illustrated by mapping its values to the covariate scales. The estimated probability of late CS after admission was 0.352 (Figure 2B).

\section{Performance of the LASSO risk nomogram model}

In this cohort, the nomogram calibration curve for the prediction of CS risk in STEMI patients demonstrated good agreement (Figure $3 A$ ). The C-index for the prediction nomogram was 0.811 (95\% CI: 0.769-0.853) for the cohort and 0.821 following bootstrapping validation, indicating intense discrimination by the model. The output of the LASSO nomogram model thus indicated a strong predictive ability. The AUCs calculated for each of the risk models for late CS following admission are shown in Figure 3B. The highest performance of the LASSO risk model demonstrated its improved predictive accuracy (AUC $=0.811)$ over the simple-ORBI model (based only on admission variables in the ORBI score; $\mathrm{AUC}=0.756$ ), a 
Table 1 Characteristics of the patients in the training and test datasets

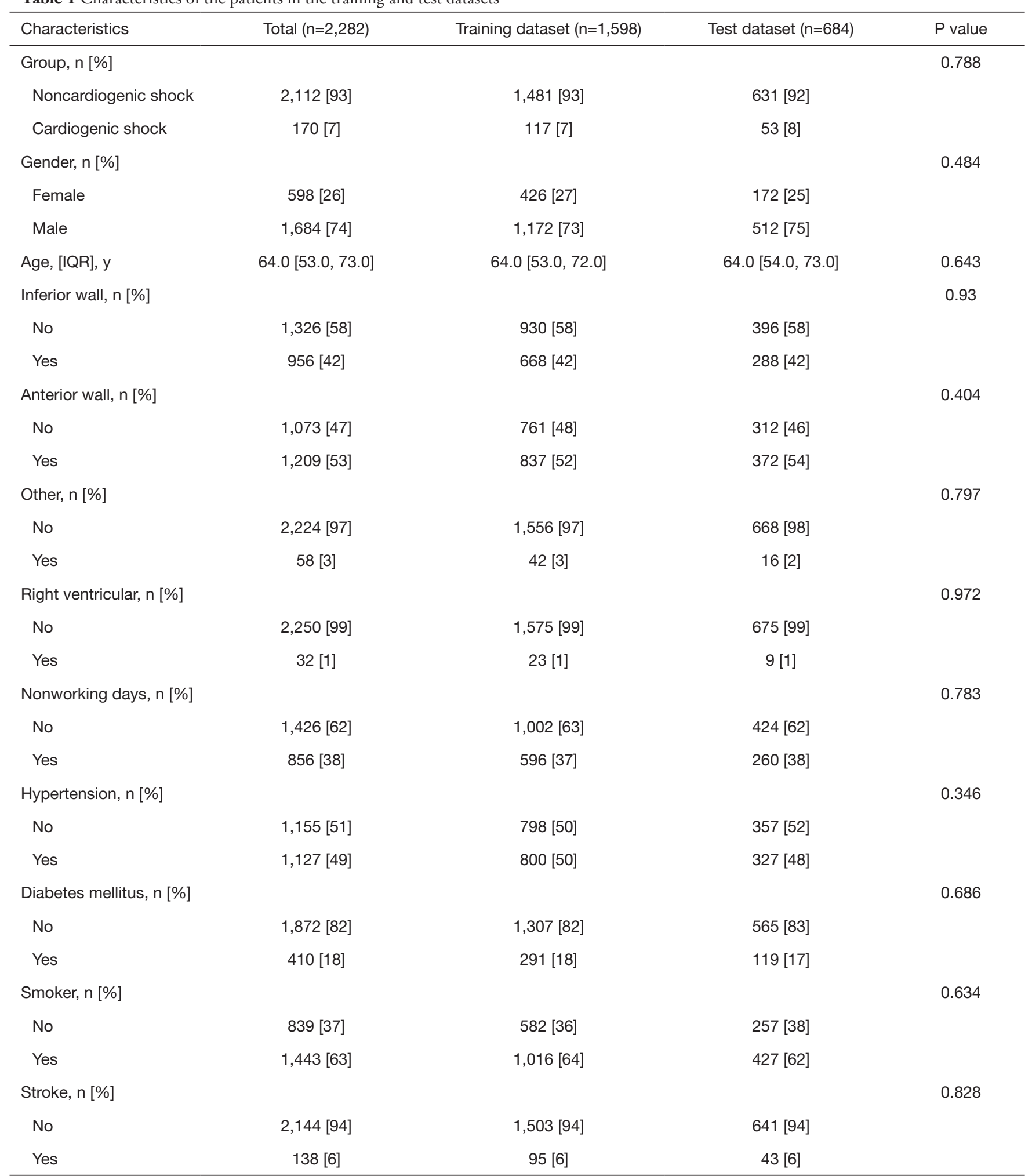

Table 1 (continued) 
Table 1 (continued)

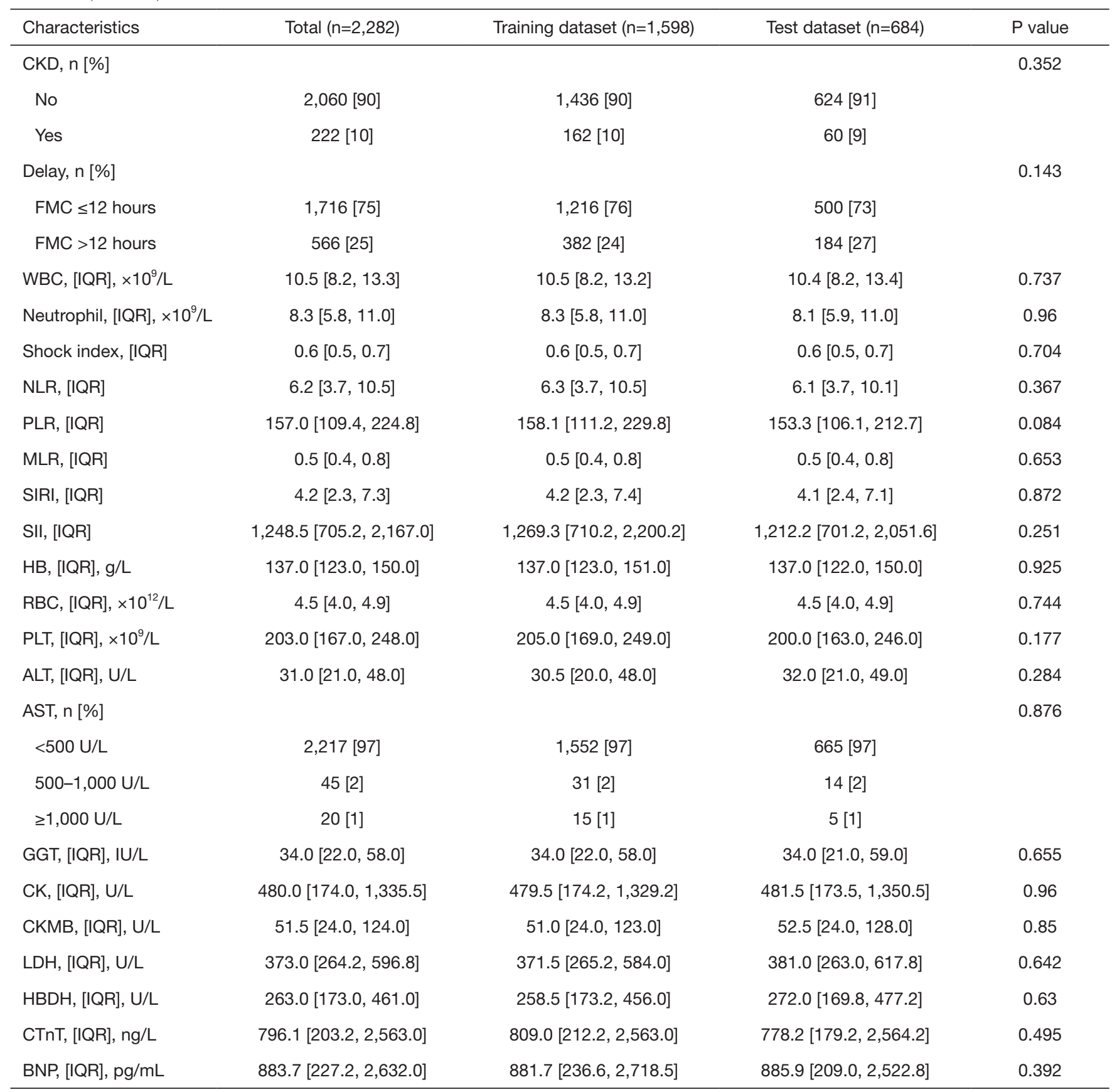

Values are expressed as medians with IQR for continuous data. Other values are presented as numbers and percentages. CKD, chronic kidney disease; FMC, first medical contact; WBC, white blood cell; Shock index, ratio of HR to SBP; PLR, ratio of platelets to lymphocytes; NLR, ratio of neutrophils to lymphocytes; MLR, ratio of monocytes to lymphocytes; SIRI, systemic inflammatory response index; SII, systemic inflammatory reaction index; HB, hemoglobin; RBC, red blood cell; PLT, platelet; ALT, alanine aminotransferase; AST, aspartate aminotransferase; GGT, gamma glutamyl transferase; CK, creatine kinase; CKMB, creatine kinase MB; LDH, lactate dehydrogenase; $\mathrm{HBDH}$, hydroxybutyrate dehydrogenase; CTnT, cardiac troponin T; BNP, brain natriuretic peptide; IQR, interquartile ranges. 
Table 2 A comparison of the model performances with the training and test datasets

\begin{tabular}{|c|c|c|c|c|c|c|c|c|c|c|}
\hline Model & \multicolumn{5}{|c|}{ Training dataset } & \multicolumn{5}{|c|}{ Test dataset } \\
\hline LR & 0.934 & 0.826 & 0.203 & 0.686 & 0.653 & 0.937 & 0.823 & 0.308 & 0.696 & 0.645 \\
\hline LASSO & 0.927 & 0.803 & 0.085 & 0.556 & 0.605 & 0.931 & 0.822 & 0.212 & 0.647 & 0.643 \\
\hline XGBoost & 1 & 1 & 1 & 1 & 1 & 0.927 & 0.782 & 0.25 & 0.541 & 0.566 \\
\hline SVM & 0.928 & 0.767 & 0.042 & 0.833 & 0.535 & 0.927 & 0.778 & 0.077 & 0.667 & 0.557 \\
\hline
\end{tabular}

LR, logistic regression; LASSO, least absolute shrinkage and selection operator; XGBoost, extreme gradient boosting; LightGBM, light gradient boosting machine; SVM, support vector regression; Acc, accuracy; AUC, area under the curve.

model based on GRACE (AUC $=0.778$ ), and a model based on the admission SI (AUC $=0.645$ ). The prediction model was further evaluated by DCA and the clinical impact curve (CIC; Figure 3C,D). The results demonstrated that the LASSO model provided a substantial net clinical benefit over the simple-ORBI model, GRACE model, and the admission SI model for relevant decision thresholds. For a decision threshold of $10 \%$ in-hospital CS risk, the LASSO nomogram model identified 20 additional cases compared to the other models, without identifying any false positive cases in a population of 1,000 patients.

\section{Discussion}

As the prognosis in patients with CS is grave and evidencebased treatment is limited among patients with fulminant CS, the identification of patients in a pre-CS state may be important (14). In this study, five machine learning models were used to predict CS risk in STEMI patients. Although LightGBM and XGBoost showed the highest AUC and accuracy in the training dataset, those in the test dataset are the most important for constructing a predictive model for an early warning system in clinical practice. Among the models constructed, the LASSO method (accuracy $=0.931, \mathrm{AUC}=0.822$, recall $=0.212$, precision $=0.647$, Gini coefficient $=0.643$ ) achieved the best performance for CS prediction with the test dataset (Table 2, Figure S2). The LASSO model had a stronger explanatory ability and applicability than the other models. LASSO regression results in a full shrinkage of a subset of variables, which effectively operates as a form of variable selection and results in a more stable model that produces a better predictor, particularly when applied to external datasets (15). To date, nomograms have been widely used in the prognostic analysis of tumors and other medical conditions (16). Nomograms rely on a user-friendly digital interface, high accuracy, and a clear interpretation of prognosis to aid clinical decision-making (17). This study is the first to evaluate the efficacy of of multiple machine learning models and select the optimal model with which to construct a nomogram and apply it to the risk prediction of STEMI patients developing late CS following admission.

In this sizeable homogeneous cohort of STEMI patients without CS on admission, we developed and validated a new predictive tool that uses 8 variables, including 4 patientrelated variables and 4 laboratory-related variables, to predict the development of in-hospital CS after admission. Integrating risk factors for age, disease, and laboratory measurements into an easy-to-use nomogram helps the individualized prediction of late CS development in patients with STEMI via the creation of a risk nomogram. The internal validation of the model showed good differentiation and calibration capability. In particular, our high C-index from the internal validation indicated that the nomogram can be used extensively and accurately because of the reasonably large sample size used in its construction. In agreement with previous studies (18-20), approximately $5-10 \%$ of STEMI patients in this study presented with CS. Recent analyses indicated that older age, diabetes mellitus, stroke, treatment delays, anterior STEMI, heart rate, systolic blood pressure, cardiac arrest, elevated glycemia, and impaired renal function were associated with the development of in-hospital CS (14,21-24). In this current study, the risk factors for CS in STEMI patients were determined to be age, CKD, SI, delay, and laboratory measurements including $\mathrm{WBC}, \mathrm{HB}, \mathrm{AST}$, and LDH. Moreover, in the recently published ORBI risk score study, which included 9,046 STEMI patients, older age, SI, and longer delays between symptoms were also predictors 

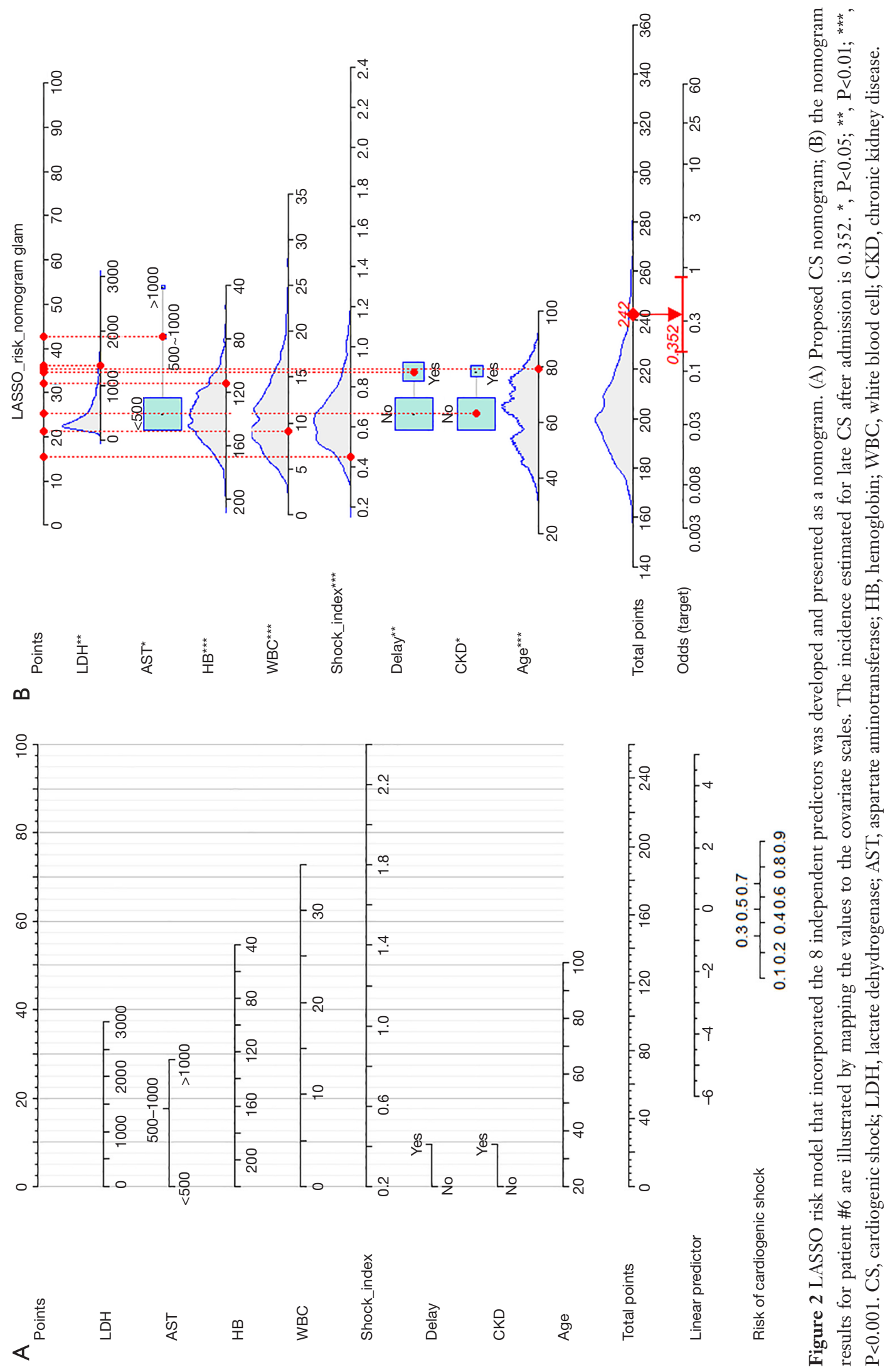
A

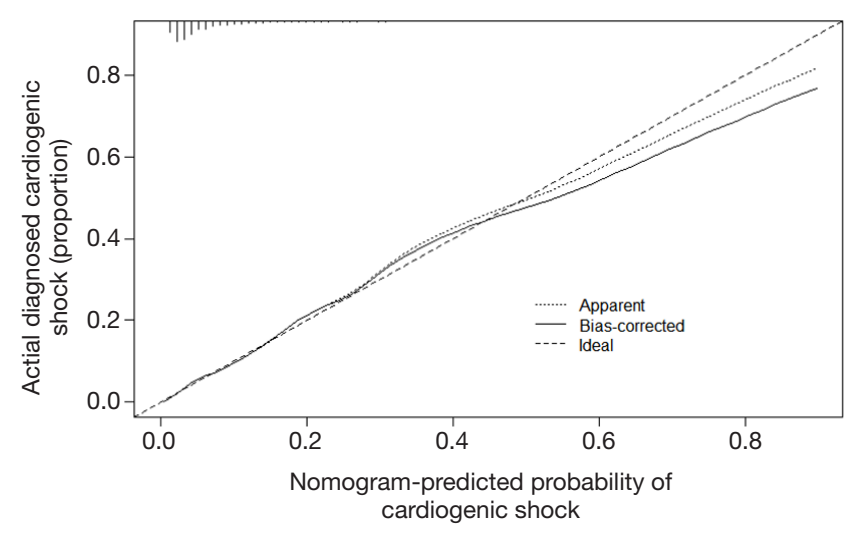

C



B

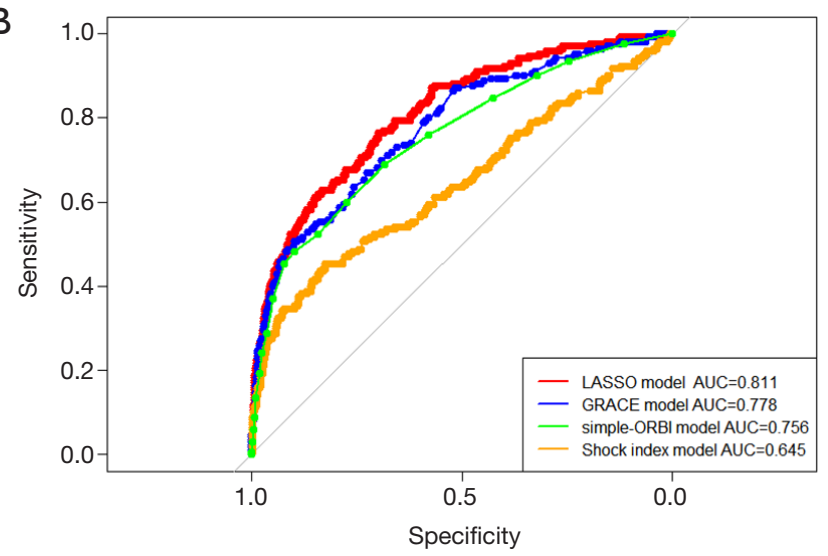

D



Figure 3 Performance of the LASSO risk nomogram model. (A) Calibration curves for the predictions of the CS nomogram in the cohort. The solid line represents the performance of the nomogram; a closer fit to the diagonal dotted line represents a better prediction. (B) Predictive accuracy of the LASSO model, GRACE model, simple-ORBI model (based only on admission variables in the ORBI score, such as age, previous stroke/TIA, presentation with cardiac arrest, anterior myocardial infarction, FMC delay >90 min, Killip Class II or III, heart rate $>90$ beats $/ \mathrm{min}$, and the combination of systolic blood pressure $<125 \mathrm{mmHg}$ and pulse pressure $<45 \mathrm{mmHg}$ ) and shock index model for late CS. (C) Decision curve analysis for the CS nomogram. The LASSO nomogram model (red) demonstrated an improved net benefit compared with the simple-ORBI model (green), the GRACE model (blue), and a model based on admission shock index (orange). (D) Clinical impact curve for the LASSO nomogram model. The heavy red solid line shows the total number of patients out of 1,000 who would be deemed high risk for each risk threshold. The blue dashed line shows how many of those patients would be true positive cases. CS, cardiogenic shock; LASSO, least absolute shrinkage and selection operator; GRACE, Global Registry of Acute Coronary Events; ORBI, Brittany Regional Infarction Observatory; TIA, transient ischemic attack; FMC, first medical contact.

of CS, which concurs with the prognostic impact of these factors identified in the present analysis.

There is a close relationship between renal and cardiovascular diseases, which may be related to the shared profile of risk factors (25). Compared with normal kidney function in healthy patients, abnormal kidney function in STEMI patients is associated with a greater than $70 \%$ increase in the risk of adverse clinical events (26). Chronic renal failure has been defined as a prognostic determinant in STEMI patients complicated with CS (27). CKD is a predisposing factor for renal acidosis and since severe hypotension deteriorates renal perfusion, it exacerbates renal acidosis and plays a prominent role in the vicious cycle encountered in CS patients. Furthermore, the success of PCI has been reported to be low in patients with CKD, and their coronary atherosclerotic plaques tend to have marked 
calcification with increased media thickness, particularly in end-stage renal disease patients. In addition, microvascular impairment has been observed in patients with $\operatorname{CKD}(28,29)$. In this model, delay refers to an FMC $>12$ hours. These patients may present with atypical symptoms or a lack of awareness of their condition. At present, the patient delay is the main reason for the total excess time of STEMI ischemia and poor prognosis. Systemic delay can be improved by constructing chest pain centers and training the medical team (30). To minimize patient delay, the public should be made awareness of the common symptoms of AMI and when to call emergency services. The model presented in this study confirmed that SI is a valuable indicator for predicting prognosis in patients with AMI complicated by CS. Previous studies have used SI to predict mortality in the ACS population and have found that an increased SI can predict short-term mortality in patients with STEMI. A SI $\geq 0.7-0.8$ on admission in patients with STEMI was associated with a mortality rate of $16-20 \%$, and the lower the SI, the lower the risk of death (31-34). This study showed that the traditional inflammatory indicators, WBC count and myocardial enzymes (LDH and AST), remain essential in the risk prediction of STEMI and confirmed that anemia might increase the risk among patients after PCI. Pathophysiologically, hemoglobin concentration and blood oxygen saturation decrease in patients with anemia, which leads to an imbalance in the oxygen supply, consumption of cardiomyocytes, and myocardial ischemia. After myocardial ischemia, a reflex causes the activation of the sympathetic nerve and the angiotensin aldosterone system, which leads to a compensatory increase in heart rate and blood volume. This increase leads to an increase in cardiac load and aggravates myocardial ischemia. In addition, vascular inflammation increases in patients with anemia, which can aggravate coronary artery plaques and thrombi.

The machine learning-based nomogram presented in this study has several strengths. First, the nomogram was developed from a largely homogeneous population of STEMI patients analyzed by machine learning with improved model performance over traditional regression methods. This method also results in a superior final prediction model without sacrificing the interpretability of the relationship between risk factors and the outcomes of interest. Second, the assessment of the risk of bias (ROB) is an essential step in any prediction model study. A high ROB will overestimate the model efficacy. The Prediction Model Risk of Bias Assessment Tool (PROBAST) was developed to address the lack of appropriate tools specific to the evaluation of $\mathrm{ROB}$ and the applicability of prediction model studies (35). The ROB is low when predictions are made without knowledge of the outcome status. Based on the patient's clinical and laboratory data on admission, the proposed LASSO risk model of late CS was constructed to ensure a low ROB. Additionally, there was no risk of delayed calculation caused by waiting for the procedural results to be available. Third, as previously discussed, to ensure that the outcome was isolated to the predictors, several known risk factors for the development of CS in STEMI were not included in the present study, such as in-hospital treatment (including PCI and coronary artery bypass grafting), diagnostic variables, left ventricular ejection fraction (LVEF), and lactate levels. The inclusion of these factors would have inflated the apparent model performance because they are measured closer in time to the outcome assessment and are likely to be more strongly associated with the outcome. Fourth, the nomogram demonstrated superior clinical benefit compared with the simple-ORBI model (based on ORBI but excluding procedural variables) and the GRACE model, which have been externally validated in the general population for predicting in-hospital late CS $(9,10)$. Finally, the nomogram efficiently addresses a significant clinical need, refining the identification of patients at high risk of CS development on admission, which may be the most suitable time to initiate early adjunctive therapies. This was the first machine learning model developed for this condition using a large sample size, and the results were displayed directly via a nomogram, which can be easily applied in clinical practice. The early prediction of CS has positive clinical importance in the treatment and prognosis of patients with STEMI. Patients at higher risk may benefit from early management, which may prevent iatrogenic shock.

\section{Limitations}

There were certain limitations to this current research. First, the data collected only represented a portion of STEMI patients and may not be representative of all STEMI patients. Second, not all possible factors that influence CS were included in the risk factor analyses. For example, glycemia is not routinely recorded in our patients and therefore could not be tested as a potential predictor of CS. Additionally, the influence of other variables, such as insurance and other factors, are not fully understood. Third, although the bootstrap test thoroughly 
assessed the robustness of our nomogram, external testing could not be conducted, and the generalizability to other STEMI populations in other regions and countries is unclear. Further investigations using larger populations are warranted to fully evaluate the applicability of this model.

\section{Conclusions}

This research established a new nomogram capable of predicting the risk of CS in STEMI patients with good accuracy. This nomogram is a simple and efficient tool that may be implemented on admission in routine clinical practice to identify STEMI patients with a high risk of developing in-hospital CS.

\section{Acknowledgments}

Funding: None.

\section{Footnote}

Reporting Checklist: The authors have completed the TRIPOD reporting checklist. Available at https://dx.doi. org/10.21037/atm-21-2905

Data Sharing Statement: Available at https://dx.doi. org/10.21037/atm-21-2905

Conflicts of Interest: All authors have completed the ICMJE uniform disclosure form (available at https://dx.doi. org/10.21037/atm-21-2905). The authors have no conflicts of interest to declare.

Ethical Statement: The authors are accountable for all aspects of the work in ensuring that questions related to the accuracy or integrity of any part of the work are appropriately investigated and resolved. This study was locally approved by the Ethics Committee of the Affiliated Hospital of Zunyi Medical University (approval No. KLL[2020]0144). All procedures performed in this study involving human participants were in accordance with the Declaration of Helsinki (as revised in 2013). Patient written informed consent was waived due to the retrospective nature of the study.

Open Access Statement: This is an Open Access article distributed in accordance with the Creative Commons Attribution-NonCommercial-NoDerivs 4.0 International
License (CC BY-NC-ND 4.0), which permits the noncommercial replication and distribution of the article with the strict proviso that no changes or edits are made and the original work is properly cited (including links to both the formal publication through the relevant DOI and the license). See: https://creativecommons.org/licenses/by-nc-nd/4.0/.

\section{References}

1. Combes A, Price S, Slutsky AS, et al. Temporary circulatory support for cardiogenic shock. Lancet 2020;396:199-212.

2. Nguyen HL, Yarzebski J, Lessard D, et al. Ten-Year (20012011) Trends in the Incidence Rates and Short-Term Outcomes of Early Versus Late Onset Cardiogenic Shock After Hospitalization for Acute Myocardial Infarction. J Am Heart Assoc 2017;6:e005566.

3. Mathias W, Tsutsui JM, Tavares BG, et al. Sonothrombolysis in ST-Segment Elevation Myocardial Infarction Treated With Primary Percutaneous Coronary Intervention. J Am Coll Cardiol 2019;73:2832-42.

4. Schrage B, Ibrahim K, Loehn T, et al. Impella Support for Acute Myocardial Infarction Complicated by Cardiogenic Shock. Circulation 2019;139:1249-58.

5. Du X, Patel A, Anderson CS, et al. Epidemiology of Cardiovascular Disease in China and Opportunities for Improvement: JACC International. J Am Coll Cardiol 2019;73:3135-47.

6. Thiele H, Akin I, Sandri M, et al. One-Year Outcomes after PCI Strategies in Cardiogenic Shock. N Engl J Med 2018;379:1699-710.

7. Thiele H, Ohman EM, de Waha-Thiele S, et al. Management of cardiogenic shock complicating myocardial infarction: an update 2019. Eur Heart J 2019;40:2671-83.

8. Ibanez B, James S, Agewall S, et al. 2017 ESC Guidelines for the management of acute myocardial infarction in patients presenting with ST-segment elevation: The Task Force for the management of acute myocardial infarction in patients presenting with ST-segment elevation of the European Society of Cardiology (ESC). Eur Heart J 2018;39:119-77.

9. Kao YT, Hsieh YC, Hsu CY, et al. Comparison of the TIMI, GRACE, PAMI and CADILLAC risk scores for prediction of long-term cardiovascular outcomes in Taiwanese diabetic patients with ST-segment elevation myocardial infarction: From the registry of the Taiwan Society of Cardiology. PLoS One 2020;15:e0229186. 
10. Auffret V, Cottin Y, Leurent G, et al. Predicting the development of in-hospital cardiogenic shock in patients with ST-segment elevation myocardial infarction treated by primary percutaneous coronary intervention: the ORBI risk score. Eur Heart J 2018;39:2090-102.

11. Collins GS, Reitsma JB, Altman DG, et al. Transparent reporting of a multivariable prediction model for individual prognosis or diagnosis (TRIPOD): the TRIPOD statement. The TRIPOD Group. Circulation 2015;131:211-9.

12. Kawaji T, Shiomi H, Morimoto T, et al. Long-term clinical outcomes in patients with ST-segment elevation acute myocardial infarction complicated by cardiogenic shock due to acute pump failure. Eur Heart J Acute Cardiovasc Care 2018;7:743-54.

13. Levey AS, Stevens LA, Schmid CH, et al. A new equation to estimate glomerular filtration rate. Ann Intern Med 2009;150:604-12.

14. Quenot JP, Jacquier M, Dargent A, et al. Preemptive renal replacement therapy in post-cardiotomy cardiogenic shock patients: a new concept? Ann Transl Med 2020;8:613.

15. Goldstein BA, Navar AM, Carter RE. Moving beyond regression techniques in cardiovascular risk prediction: applying machine learning to address analytic challenges. Eur Heart J 2017;38:1805-14.

16. Wang H, Zhang L, Liu Z, et al. Predicting medication nonadherence risk in a Chinese inflammatory rheumatic disease population: development and assessment of a new predictive nomogram. Patient Prefer Adherence 2018;12:1757-65.

17. Wei L, Champman S, Li X, et al. Beliefs about medicines and non-adherence in patients with stroke, diabetes mellitus and rheumatoid arthritis: a cross-sectional study in China. Bmj Open 2017;7:e017293.

18. Damluji AA, Bandeen-Roche K, Berkower C, et al. Percutaneous Coronary Intervention in Older Patients With ST-Segment Elevation Myocardial Infarction and Cardiogenic Shock. J Am Coll Cardiol 2019;73:1890-900.

19. Vahdatpour C, Collins D, Goldberg S. Cardiogenic Shock. J Am Heart Assoc 2019;8:e011991.

20. Khan MZ, Munir MB, Khan MU, et al. Trends, Outcomes, and Predictors of Revascularization in Cardiogenic Shock. Am J Cardiol 2020;125:328-35.

21. Goldberg RJ, Makam RC, Yarzebski J, et al. Decade-Long Trends (2001-2011) in the Incidence and Hospital Death Rates Associated with the In-Hospital Development of Cardiogenic Shock after Acute Myocardial Infarction. Circ Cardiovasc Qual Outcomes 2016;9:117-25.
22. Toleva O, Ibrahim Q, Brass N, et al. Treatment choices in elderly patients with ST: elevation myocardial infarctioninsights from the Vital Heart Response registry. Open Heart 2015;2:e000235.

23. Sulo E, Vollset SE, Nygard O, et al. Trends in 28-day and 1 -year mortality rates in patients hospitalized for a first acute myocardial infarction in Norway during 2001-2009: a "Cardiovascular disease in Norway" (CVDNOR) project. J Intern Med 2015;277:353-61.

24. Weipert KF, Bauer T, Nef HM, et al. Incidence and outcome of peri-procedural cardiogenic shock: results from the international Euro Heart Survey PCI registry. Eur Heart J Acute Cardiovasc Care 2020;9:120-7.

25. Said S, Hernandez GT. The link between chronic kidney disease and cardiovascular disease. J Nephropathol 2014;3:99-104.

26. Alkhalil M, Mccune C, Mcclenaghan L, et al. Comparative Analysis of the Effect of Renal Function on the Spectrum of Coronary Artery Disease. Am J Med 2020;133:e631-e640.

27. Hayıroğlu Mİ, Keskin M, Uzun AO, et al. Predictors of In-Hospital Mortality in Patients With ST-Segment Elevation Myocardial Infarction Complicated With Cardiogenic Shock. Heart Lung Circ 2019;28:237-44.

28. Costanzo P, Dzavik V. Coronary Revascularization in Patients With Advanced Chronic Kidney Disease. Can J Cardiol 2019;35:1002-14.

29. Tebaldi M, Biscaglia S, Fineschi M, et al. Fractional Flow Reserve Evaluation and Chronic Kidney Disease: Analysis From a Multicenter Italian Registry (the FREAK Study). Catheter Cardiovasc Interv 2016;88:555-62.

30. Fordyce CB, Al-Khalidi HR, Jollis JG, et al. Association of Rapid Care Process Implementation on Reperfusion Times Across Multiple ST-Segment-Elevation Myocardial Infarction Networks. Circ Cardiovasc Interv 2017;10:e004061.

31. Huang B, Yang Y, Zhu J, et al. Usefulness of the admission shock index for predicting short-term outcomes in patients with ST-segment elevation myocardial infarction. Am J Cardiol 2014;114:1315-21.

32. Bilkova D, Motovska Z, Widimsky P, et al. Shock index: a simple clinical parameter for quick mortality risk assessment in acute myocardial infarction. Can J Cardiol 2011;27:739-42.

33. Spyridopoulos I, Noman A, Ahmed JM, et al. Shockindex as a novel predictor of long-term outcome following primary percutaneous coronary intervention. Eur Heart J Acute Cardiovasc Care 2015;4:270-7. 
34. Supeł K, Kacprzak M, Zielinska M. Shock index and TIMI risk index as valuable prognostic tools in patients with acute coronary syndrome complicated by cardiogenic shock. PLoS One 2020;15:e0227374.

Cite this article as: Bai Z, Hu S, Wang Y, Deng W, Gu N, Zhao R, Zhang W, Ma Y, Wang Z, Liu Z, Shen C, Shi B. Development of a machine learning model to predict the risk of late cardiogenic shock in patients with ST-segment elevation myocardial infarction. Ann Transl Med 2021;9(14):1162. doi: 10.21037/atm-21-2905
35. Moons KG, Wolff RF, Riley RD, et al. PROBAST: A Tool to Assess Risk of Bias and Applicability of Prediction Model Studies: Explanation and Elaboration. Ann Intern Med 2019;170:W1-W33. 


\section{Performance Metrics}

In the current study, the following metrics were applied to evaluate the performance of each model.

i.) True positives (TPs), positive diagnoses classified as positive outcomes.

ii.) False positives (FPs), negative diagnoses classified as positive outcomes.

iii.) True negatives ( $\mathrm{TNs}$ ), negative diagnoses classified as negative outcomes.

iv.) False negatives (FNs), positive diagnoses classified as negative outcomes.

v.) The learning curve, adopted to assess classification performance (Figure S3).

vi.) Accuracy, the ability to correctly classify the dataset

Accuracy $=\frac{T P+T N}{T P+F P+T N+F N}$

vii.) True positive rate (TPR), or Sensitivity

$T P R=$ Recall $=$ Sensitivity $=\frac{T P}{T P+F N}$

viii.) False positive rate (FPR), or type I error probability

$F P R=F P /(F P+T N)$

ix.) True negative rate (TNR), or Specificity

Specificity $=T N R=\frac{T N}{T N+F P}=1-F P R$

x.) Precision

Precision $=\frac{T P}{T P+F P}$

xi.) Receiver operating characteristic (ROC) curve, a curve determined by plotting TPR and FPR and used for evaluating the model performance.

xii.) Area under the curve (AUC), an index used to evaluate the predictive and classification performance of a model.

xiii.) Gini coefficient, used to measure the performance of a model.

Gini $=2 * A U C-1$ 


\begin{tabular}{|c|c|c|c|c|}
\hline Variables & Total $(n=2282)$ & $\begin{array}{c}\text { Noncardiogenic shock } \\
(\mathrm{n}=2,112)\end{array}$ & Cardiogenic shock $(n=170)$ & $P$ value \\
\hline \multicolumn{5}{|l|}{ Demographic } \\
\hline Age, median (IQR) & $64.0(53.0,73.0)$ & $64.0(53.0,72.0)$ & $70.0(62.2,77.0)$ & $<0.001$ \\
\hline Sex, n (\%) & & & & $<0.001$ \\
\hline Female & $598(26)$ & $534(25)$ & $64(38)$ & \\
\hline Male & $1684(74)$ & $1578(75)$ & $106(62)$ & \\
\hline Smoker, n (\%) & & & & 0.021 \\
\hline No & $839(37)$ & $762(36)$ & $77(45)$ & \\
\hline Yes & $1443(63)$ & $1350(64)$ & $93(55)$ & \\
\hline NWD on admission, $n(\%)$ & & & & 0.109 \\
\hline No & $1426(62)$ & $1330(63)$ & $96(56)$ & \\
\hline Yes & $856(38)$ & $782(37)$ & $74(44)$ & \\
\hline Delay, n (\%) & & & & $<0.001$ \\
\hline$F M C \geq 12$ hours & $1716(75)$ & $1612(76)$ & $104(61)$ & \\
\hline FMC $<12$ hours & $566(25)$ & $500(24)$ & $66(39)$ & \\
\hline \multicolumn{5}{|l|}{ Electrocardiographic data } \\
\hline \multicolumn{5}{|l|}{ Inferior wall, n (\%) } \\
\hline No & $1326(58)$ & $1221(58)$ & $105(62)$ & \\
\hline Yes & $956(42)$ & $891(42)$ & $65(38)$ & \\
\hline Anterior wall, n (\%) & & & & 0.304 \\
\hline No & $1073(47)$ & $1000(47)$ & $73(43)$ & \\
\hline Yes & $1209(53)$ & $1112(53)$ & $97(57)$ & \\
\hline Right ventricular, $\mathrm{n}(\%)$ & & & & 0.51 \\
\hline No & 2250 (99) & 2081 (99) & $169(99)$ & \\
\hline Yes & 32 (1) & 31 (1) & $1(1)$ & \\
\hline Other, $\mathrm{n}(\%)$ & & & & 0.009 \\
\hline No & $2224(97)$ & $2064(98)$ & $160(94)$ & \\
\hline Yes & 58 (3) & 48 (2) & 10 (6) & \\
\hline \multicolumn{5}{|l|}{ Medical history } \\
\hline Hypertension, n (\%) & & & & 0.469 \\
\hline No & $1155(51)$ & $1074(51)$ & $81(48)$ & \\
\hline Yes & $1127(49)$ & $1038(49)$ & $89(52)$ & \\
\hline Diabetes mellitus, $n$ (\%) & & & & 0.216 \\
\hline No & $1872(82)$ & $1739(82)$ & $133(78)$ & \\
\hline Yes & $410(18)$ & $373(18)$ & 37 (22) & \\
\hline Stroke, n (\%) & & & & 0.941 \\
\hline No & 2144 (94) & 1985 (94) & $159(94)$ & \\
\hline Yes & $138(6)$ & 127 (6) & 11 (6) & \\
\hline CKD, n (\%) & & & & $<0.001$ \\
\hline No & $2060(90)$ & $1926(91)$ & $134(79)$ & \\
\hline Yes & $222(10)$ & $186(9)$ & $36(21)$ & \\
\hline \multicolumn{5}{|l|}{ Vital signs on admission } \\
\hline Shock index, median (IQR) & $0.6(0.5,0.7)$ & $0.6(0.5,0.7)$ & $0.7(0.6,1.0)$ & $<0.001$ \\
\hline $\mathrm{HR}$, median (IQR), beats/min & $80.0(71.0,90.0)$ & $79.0(71.0,90.0)$ & $86.0(74.2,105.0)$ & $<0.002$ \\
\hline $\mathrm{SBP}$, median (IQR), mmHg & $126.0(110.0,140.0)$ & $126.0(111.0,140.0)$ & $115.0(99.0,136.0)$ & $<0.003$ \\
\hline DBP, median (IQR), mmHg & $80.0(70.0,90.0)$ & $80.0(70.0,90.0)$ & $75.5(63.0,86.0)$ & $<0.004$ \\
\hline \multicolumn{5}{|l|}{ Laboratory on admission } \\
\hline WBC, median (IQR), ×109/L & $10.5(8.2,13.3)$ & $10.4(8.0,13.1)$ & $12.4(9.5,16.4)$ & $<0.001$ \\
\hline Neutrophil count, median (IQR), ×109/L & $8.3(5.8,11.0)$ & $8.1(5.7,10.8)$ & $10.3(7.4,13.6)$ & $<0.001$ \\
\hline NLR, median (IQR) & $6.2(3.7,10.5)$ & $6.2(3.6,9.9)$ & $8.4(4.7,14.1)$ & $<0.001$ \\
\hline PLR, median (IQR) & $157.0(109.4,224.8)$ & $157.4(110.7,223.6)$ & $152.4(95.0,253.1)$ & 0.681 \\
\hline MLR, median (IQR) & $0.5(0.4,0.8)$ & $0.5(0.4,0.8)$ & $0.6(0.4,1.0)$ & $<0.001$ \\
\hline SIRI, median (IQR) & $4.2(2.3,7.3)$ & $4.0(2.3,7.0)$ & $6.0(3.5,11.2)$ & $<0.001$ \\
\hline SIII, median (IQR) & $1248.5(705.2,2167.0)$ & $1234.3(701.1,2111.0)$ & $1564.6(787.9,2814.4)$ & 0.001 \\
\hline HB, median (IQR) & $137.0(123.0,150.0)$ & $138.0(124.0,151.0)$ & $128.0(111.0,142.0)$ & $<0.001$ \\
\hline RBC, median (IQR), $\times 1012 / L$ & $4.5(4.0,4.9)$ & $4.5(4.0,4.9)$ & $4.2(3.7,4.7)$ & $<0.001$ \\
\hline PLT, median (IQR), $\times 109 / \mathrm{L}$ & $203.0(167.0,248.0)$ & $204.0(168.0,248.0)$ & $195.5(145.5,254.8)$ & 0.135 \\
\hline ALT, median (IQR), U/L & $31.0(21.0,48.0)$ & $30.5(20.0,46.0)$ & $43.5(24.0,82.0)$ & $<0.001$ \\
\hline AST, n (\%) & & & & $<0.001$ \\
\hline$<500 \mathrm{U} / \mathrm{L}$ & $2217(97)$ & $2073(98)$ & $144(85)$ & \\
\hline $500-1000 \mathrm{U} / \mathrm{L}$ & 45 (2) & 32 (2) & $13(8)$ & \\
\hline$\geq 1000 \mathrm{U} / \mathrm{L}$ & 20 (1) & $7(0)$ & $13(8)$ & \\
\hline GGT, median (IQR), IU/L & $34.0(22.0,58.0)$ & $34.0(22.0,56.0)$ & $44.5(23.0,78.2)$ & 0.001 \\
\hline CK, median (IQR), U/L & $480.0(174.0,1335.5)$ & $470.0(172.0,1331.0)$ & $562.0(259.0,1350.0)$ & 0.065 \\
\hline CKMB, median (IQR),U/L & $51.5(24.0,124.0)$ & $51.0(24.0,124.0)$ & $65.0(29.0,138.0)$ & 0.036 \\
\hline LDH, median (IQR),U/L & $373.0(264.2,596.8)$ & $363.0(260.0,572.0)$ & $555.0(365.8,907.0)$ & $<0.001$ \\
\hline HBDH, median (IQR),U/L & $263.0(173.0,461.0)$ & $254.5(169.0,443.0)$ & $416.0(232.8,674.8)$ & $<0.001$ \\
\hline CTnT, median (IQR), ng/L & $796.1(203.2,2563.0)$ & $753.5(193.0,2424.5)$ & $2076.5(595.8,4178.5)$ & $<0.001$ \\
\hline BNP, median (IQR), $\mathrm{pg} / \mathrm{mL}$ & $883.7(227.2,2632.0)$ & $812.5(201.7,2335.5)$ & $4332.0(1082.5,12196.0)$ & $<0.001$ \\
\hline \multicolumn{5}{|l|}{ Risk assessment } \\
\hline GRACE score, median (IQR) & $122(102.0,142.0)$ & $119.0(102.0,139.0)$ & $160.0(128.0,193.8)$ & $<0.001$ \\
\hline Simple-ORBI, median (IQR) & $4.0(2.0,7.0)$ & $4.0(2.0,6.0)$ & $8.0(5.0,12.0)$ & $<0.001$ \\
\hline
\end{tabular}

Shock index ratio of HR to SBP; SIRI systemic inflammatory response index; SII systemic inflammatory reaction index; PLR ratio of platelets to lymphocytes, NLR ratio of neutrophils to lymphocytes; MLR ratio of monocytes to lymphocytes; GRACE, Global Registry of Acute Coronary Events score; $\alpha$-HBDH, $\alpha$-Hydroxybutyrate dehydrogenase; BNP B-type natriuretic peptides; NWD Non-weekday admission; CKD, Chronic kidney disease; SBP, systolic blood pressure; DBP, diastolic blood pressure; WBC, White blood cell; HB, Hemoglobin; RBC, Red blood cell; PLT, Platelet; ALT, alanine aminotransferase; AST Aspartate transaminase; GGT, glutamyl transferase, CK, creatine kinase; CKMB, creatine kinase isoenzymes; LDH, lactate dehydrogenase; CTnT, Cardiac troponin; ORBI, The Brittany Regional Infarction Observatory. 


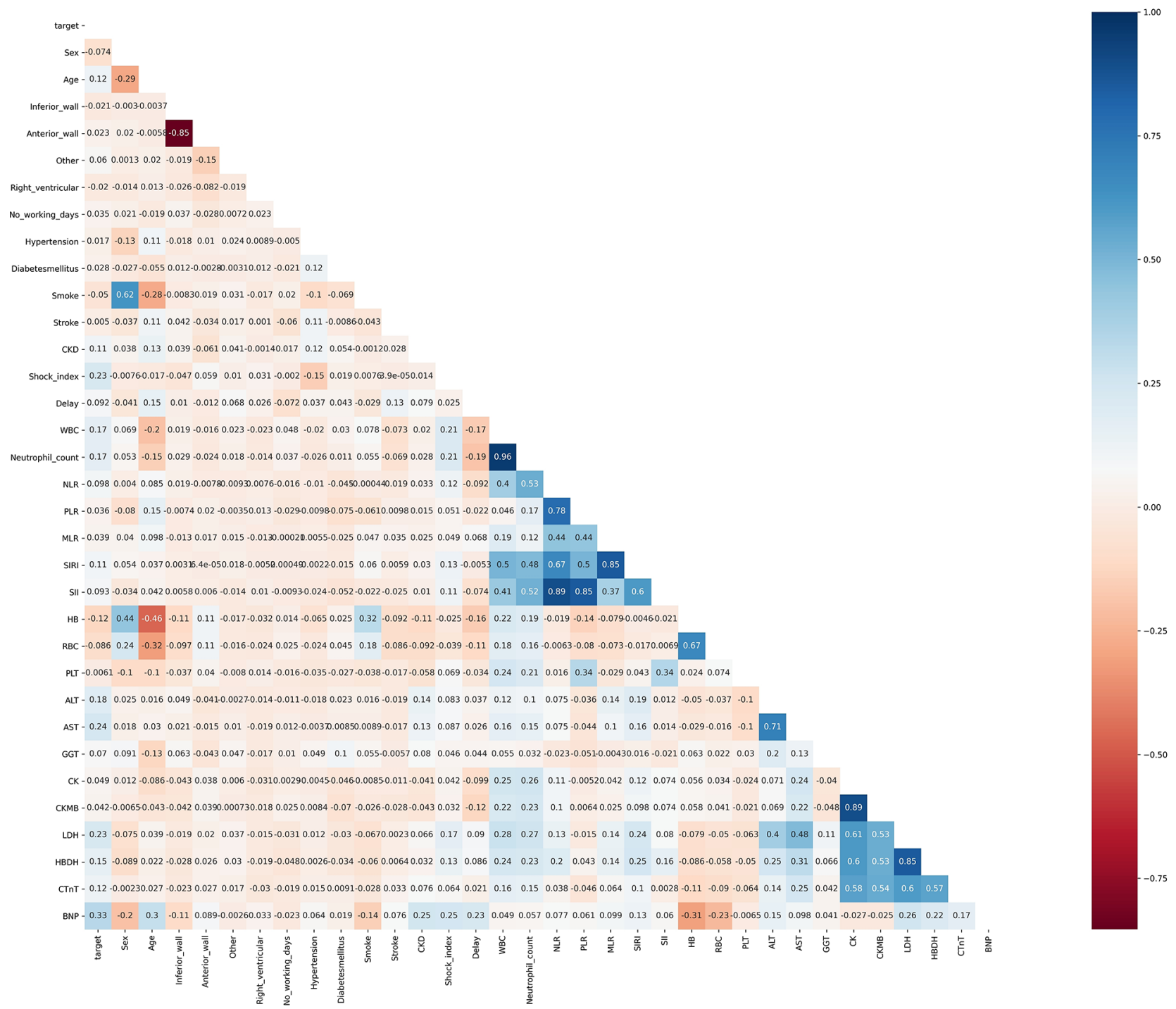

Figure S1 Variable correlation heatmap. 

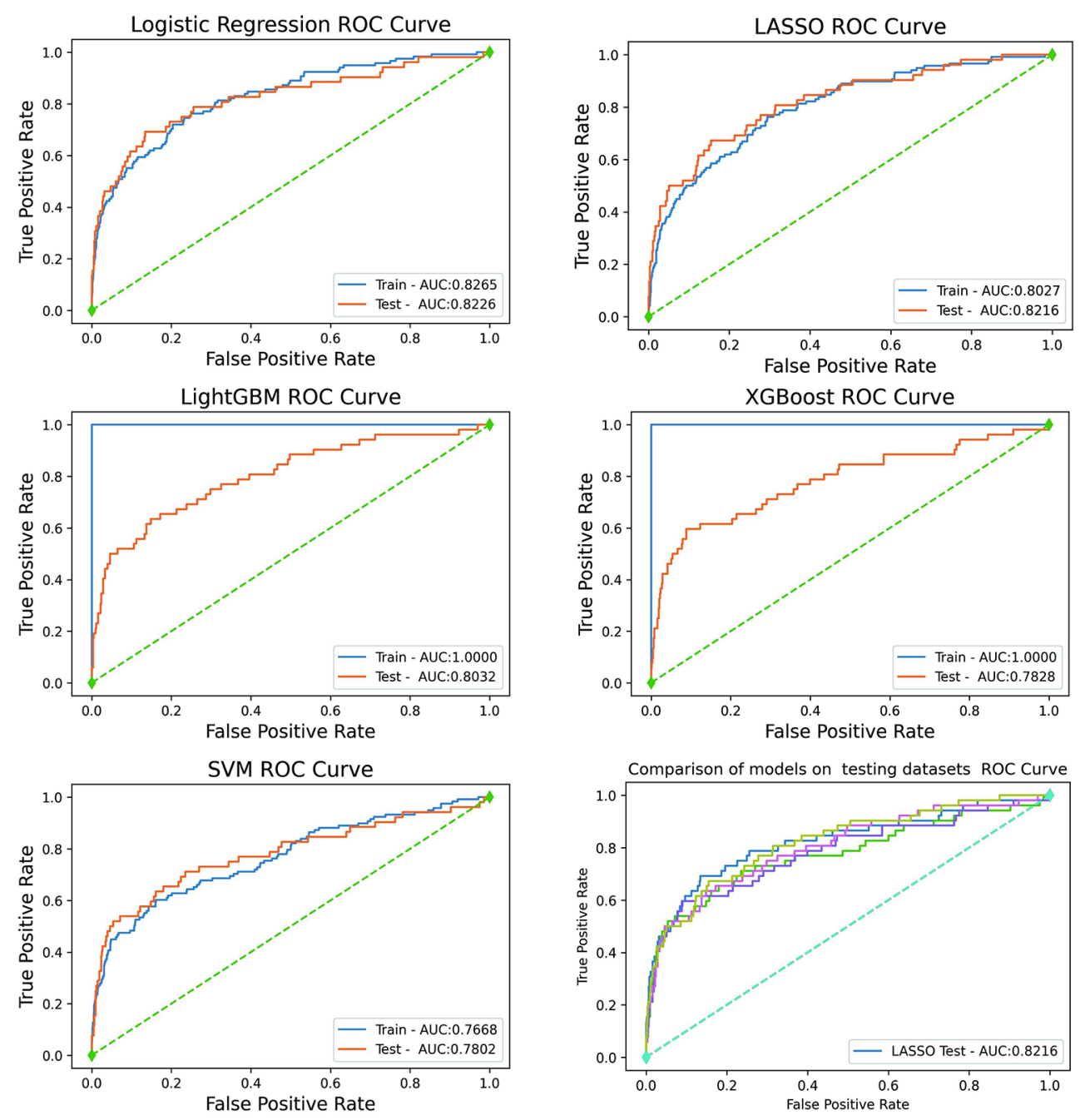

Figure S2 Receiver operating characteristic (ROC) curves of 5 machine learning model performance with the training dataset and the test dataset. 
A



C

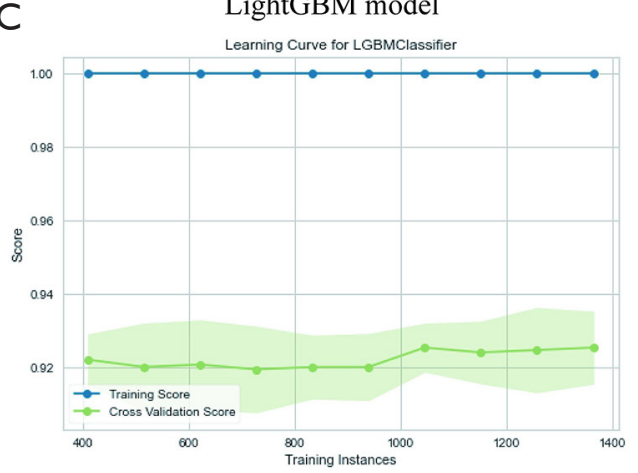

B SVM model

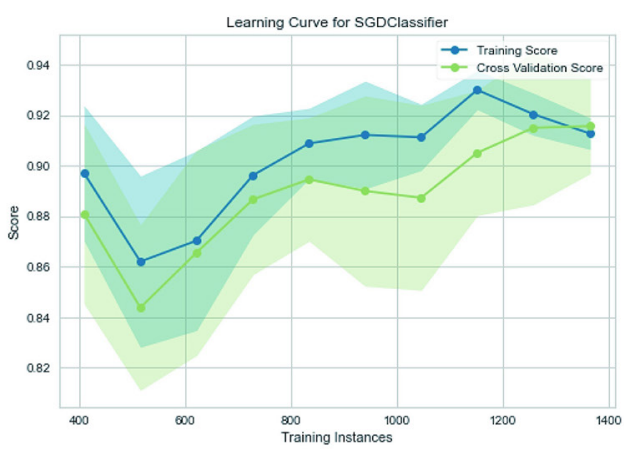

D XGBoost model

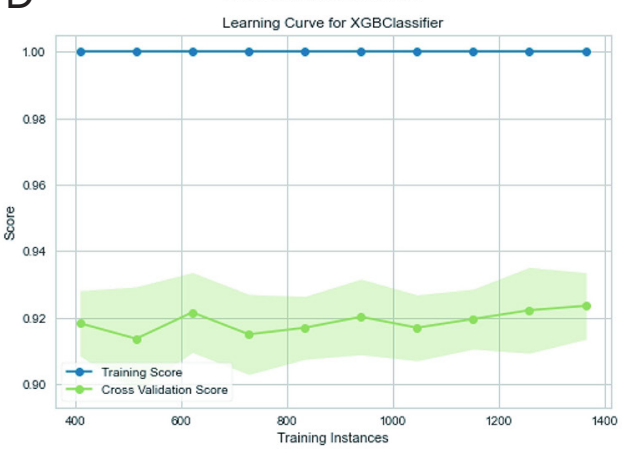

Figure S3 Learning curve for the different models.

A

\section{LR model}

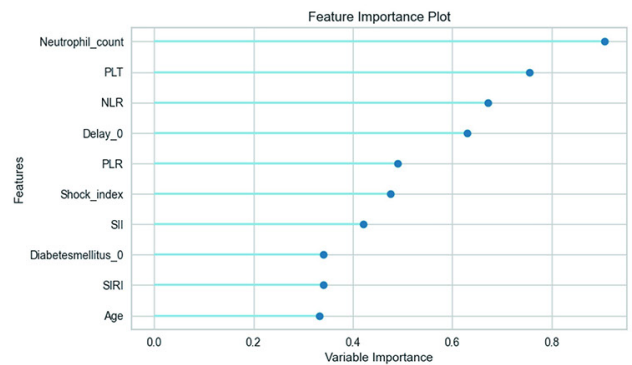

C

LightGBM model

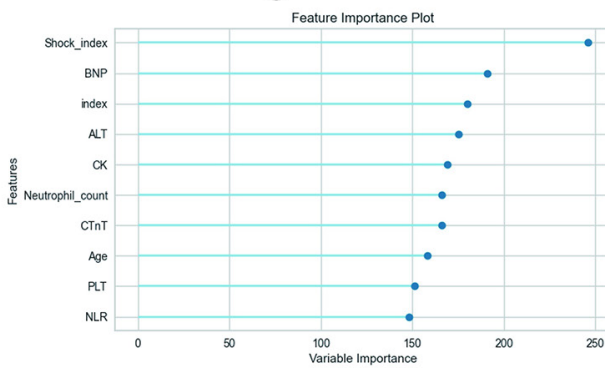

B

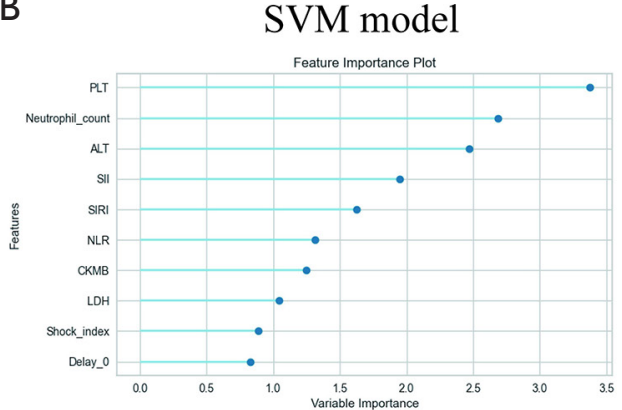

D



Figure S4 Variable importance size in the different models. 
A

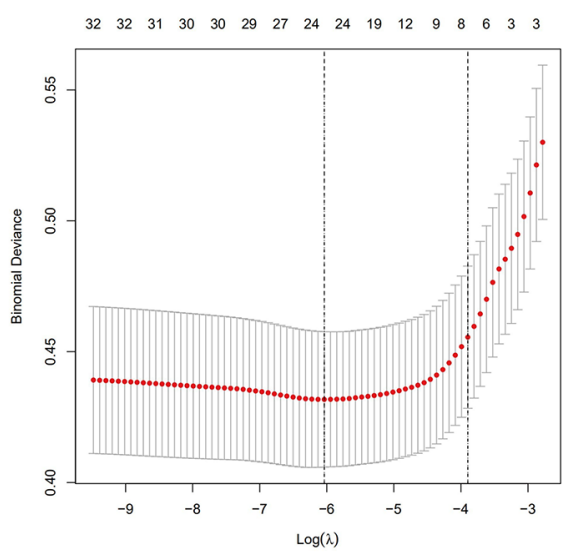

B

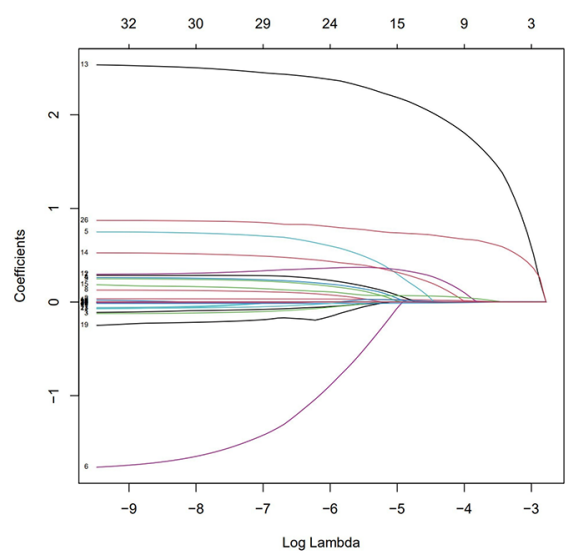

Figure S5 Selection of demographic and clinical features using the least absolute shrinkage and selection operator (LASSO) binary logistic regression model.

Table S2 Prediction factors for CS in STEMI patients

\begin{tabular}{|c|c|c|c|}
\hline \multirow{2}{*}{ Intercept and variable } & \multirow{2}{*}{$\beta$} & \multicolumn{2}{|c|}{ Prediction model } \\
\hline & & Odds ratio $(95 \% \mathrm{Cl})$ & P-value \\
\hline (Intercept) & -6.4271 & $0.0016(0.0002-0.0115)$ & $P<0.001$ \\
\hline Age & 0.0345 & $1.0351(1.0188-1.0521)$ & $P<0.001$ \\
\hline \multicolumn{4}{|l|}{ CKD } \\
\hline No & Reference & & \\
\hline Yes & 0.5254 & $1.6913(1.0504-2.6568)$ & 0.02606 \\
\hline \multicolumn{4}{|l|}{ Delay } \\
\hline No & Reference & & \\
\hline Yes & 0.5191 & $1.6805(1.1504-2.4402)$ & 0.00672 \\
\hline Shock index & 2.4828 & $11.9741(6.0138-23.9791)$ & $P<0.001$ \\
\hline WBC & 0.1137 & $1.1203(1.0769-1.1653)$ & $P<0.001$ \\
\hline HB & -0.0166 & $0.9835(0.9745-0.9926)$ & $P<0.001$ \\
\hline \multicolumn{4}{|l|}{ AST } \\
\hline$<500$ & Reference & & \\
\hline $500-1000$ & 0.9568 & $2.6032(1.1143-5.8094)$ & 0.02243 \\
\hline$>1000$ & 1.5725 & $4.8185(1.4578-16.3762)$ & 0.01005 \\
\hline LDH & 0.0007 & $1.0006(1.0002-1.0011)$ & 0.00463 \\
\hline
\end{tabular}

parameter combinations are exhausted by grid search. Performance evaluation indices such as accuracy, AUC, recall, precision and the Gini coefficient were adopted to assess the average predictive performance of the model. The optimal model was used to develop the late-CS risk nomogram. Shock index ratio of HR to SBP; WBC, white blood cell; HB, hemoglobin; CKD, chronic kidney disease; AST, aspartate transaminase; LDH, lactate dehydrogenase. 